\title{
Perfil da qualidade de vida e níveis de estresse entre mulheres praticantes de pilates
}

\author{
Profile of the quality of life and stress levels in women \\ practicing Pilates
}

\author{
Josiane Santos Oliveira ${ }^{1}$ \\ Jerri Luiz Ribeiro ${ }^{2}$ \\ Alexandre Silva de Mello ${ }^{2}$
}

\section{RESUMO}

Introdução: Qualidade de vida e estresse fazem parte de aspectos da vida diária e dependem da percepção pessoal de cada indivíduo nas situações de enfrentamento das adversidades do dia-a-dia. O objetivo deste estudo foi identificar o perfil pelos escores de mulheres praticantes de Pilates em relação ao estresse e a qualidade de vida. Métodos: Por conveniência, foi selecionado o Estúdio de Pilates Helena Rubin na cidade de Porto Alegre, com uma amostra composta de 31 mulheres entre 21 e 70 anos de idade submetidas a avaliação por meio de três questionários. Resultados: O perfil observado são mulheres casadas, meia idade, renda familiar acima de dez salários mínimos, profissionalmente ativas, com frequência de duas vezes por semana e média de 2,9 anos de prática de Pilates. A procura pela prática de Pilates foi, na maioria, uma forma terapêutica para melhorar algum acometimento em saúde que relatam melhora no vigor e disposição do dia-a-dia após iniciada a prática regular. Porém, não encontramos correlação significativa entre a idade, o tempo e a frequência da prática de Pilates em relação ao estresse e a qualidade de vida. Percebe-se que a prática de Pilates pode influenciar positivamente na qualidade de vida de seus praticantes e sugere-se que o Pilates possa agir no controle do estresse em mulheres com diversos acometimentos em saúde. Conclusão: Sendo assim, nosso estudo pode contribuir para o desenvolvimento e direcionamento de estratégias adequadas as percepções dos diferentes praticantes, principalmente, para os profissionais que buscam na qualidade do atendimento propiciar o bem estar a seus clientes.

\section{PALAVRAS-CHAVE}

Pilates, Estresse, Qualidade de vida.

\footnotetext{
${ }^{1}$ Mestra pelo Programa de Pós-Graduação em Biociências e Reabilitação do Centro Universitário Metodista - IPA.

${ }^{2}$ Docente do Centro Universitário Metodista - IPA.
} 


\section{ABSTRACT}

Introduction: Quality of life and stress are part of daily life and depend on the personal perception of each individual in situations of coping with everyday adversities. The objective of this study was to identify the profile of Pilates women in relation to stress and quality of life. Methods: For convenience, the Helena Rubin Pilates Studio was selected in the city of Porto Alegre, with a sample composed of 31 women between 21 and 70 years of age submitted to the evaluation by means of three questions. Results: The profile observed are married women, middle age, family income above ten minimum wages, professionally active, twice a week and an average of 2.9 years of Pilates practice. The search for the practice of Pilates was in the majority a therapeutic form to improve some affection in health that report improvement in the vigor and disposition of the day to day after beginning the regular practice. However, we found no significant correlation between age, time and frequency of Pilates practice in relation to stress and quality of life. It is noticed that the practice of Pilates can positively influence the quality of life of its practitioners and it is suggested that Pilates can act in the control of stress in women with diverse health concerns. Conclusion: Therefore, our study can contribute to the development and targeting of adequate strategies as perceptions of the different practitioners, mainly for the professionals who seek in the quality of the provision of welfare to their clients.

\section{KEYWORDS}

Pilates, Stress, Quality of life. 


\section{INTRODUÇÃO}

Joseph Pilates é o criador do método de exercício físico Pilates, concebido originalmente para o trabaIho de simetria postural, controle da respiração, força abdominal, estabilização de coluna vertebral, cintura escapular e pélvica, mobilidade articular, flexibilidade e fortalecimento muscular [1-4].

O termo "promoção em saúde" surge como forma de enfrentamento as condições de saúde entendidas como um estado de completo bem-estar, físico, mental e social conforme anunciado pela Organização Mundial da Saúde [5]. Nossa capacidade de resistir aos desafios do cotidiano e manter a saúde estão na dependência da percepção pessoal de cada indivíduo e refletem um comportamento positivo perante a vida que pode ser observado em como interpretamos e lidamos com o estresse e a busca por qualidade de vida $[6,7]$.

A procura pelo Pilates entre mulheres está cada vez mais relacionada ao bem estar e saúde, e faz deste método, uma importante ferramenta no desempenho independente nas atividades de vida diária (AVD's). Mulheres são mais propensas ao estresse e os efeitos benéficos do método Pilates interferem positivamente no estilo de vida [6].

Questionários são medidas de avaliação de saúde mental e física funcional, e são usados clinicamente por ser de fácil manuseio, práticos e objetivos. Estudo realizado por Natour et al. (2015) [8], com o uso do questionário de Qualidade de Vida (SF-36), resultados positivos foram observados em pacientes com lombalgia crônica não específica na qualidade de vida da amostra $[9,10]$.

Dentre os motivos que levaram a prática do método Pilates os maiores escores estão em saúde, para aptidão física, disposição, atratividade e harmonia conforme observado no estudo de Fermino et al. (2010) [11].

A rotatividade entre os praticantes é grande apesar da enorme adesão e ascensão no mercado da prática de Pilates e fidelizar os clientes, passa a ser relevante aos profissionais que trabalham com a conscientização dos seus clientes em se manterem saudáveis $[6,12]$.
A tomada de consciência dos benefícios do Pilates acarretou um olhar mais atento dos profissionais para as características de seus clientes, e se torna relevante direcionar medidas corretivas para o aperfeiçoamento no atendimento, principalmente se o objetivo do praticante for por maior qualidade de vida e controle do estresse no seu dia-a-dia [13].

A qualidade do serviço prestado deixou de ser uma ação do mercado e passou a ser tendência entre os consumidores cada vez mais exigentes com a procura e os resultados da prática de Pilates, o que desperta o interesse dos profissionais prestadores deste tipo de serviço ao entendimento do perfil deste público $[14,15]$.

Nesta perspectiva o objetivo do presente estudo foi identificar o perfil de mulheres praticantes de Pilates a fim de verificar se a prática regular estaria correlacionada com uma boa condição de saúde para os níveis de estresse e qualidade de vida.

\section{MATERIAIS E MÉTODOS}

\section{População do estudo}

Tratou-se de um estudo de coorte transversal realizado na população de mulheres frequentadoras do "Estúdio de Pilates Helena Rubin", da cidade de Porto Alegre, Rio Grande do Sul. A amostra foi composta por mulheres entre 21 e 70 anos, que não praticassem outra forma de atividade física além do Pilates, e tivessem pelo menos, dois meses de prática de forma a excluir as faltosas e irregulares.

As mulheres que atendessem ao convite verbal assinaram o Termo de Consentimento Livre e Esclarecido (TCLE) e foram esclarecidas que tratava-se de uma avaliação por meio do preenchimento de três questionários no mesmo local da prática de Pilates com um tempo estimado de trinta minutos para a avaliação.

A pesquisa seguiu os princípios éticos da Resolução 466/12 do Conselho Nacional de Saúde (CNS) com parecer $\mathrm{n}^{0}$ : 1.756 .143 , aprovado no Comitê de Ética e Pesquisa do Centro Universitário Metodista - IPA. 


\section{Instrumentos da Pesquisa}

Para coleta dos dados foram utilizados os seguintes instrumentos:

1. "Questionário da prática de Pilates", elaborado pelos autores desta pesquisa com objetivo de coletar algumas características do perfil da amostra com variáveis como idade, renda familiar, frequência, objetivos e tempo da prática de Pilates.

2. "Questionário de Qualidade de Vida", SF-36, versão para o Brasil (OMS, 1994 traduzido para o português por Fleck, 1998). As variáveis deste questionário referem-se aos domínios de saúde física e mental com perguntas fechadas.

3. “Inventário de Sintomas de Estresse para Adultos de LIPP" (ISSL) [16] é um questionário fechado que descreve questões em relação: a) existência do estresse; b) fase específica; c) prevalência de sintomas físicos ou psicológicos que investigam em três momentos da vida: nas últimas 24 horas, na última semana e no último mês.

\section{Análise dos dados}

A análise estatística selecionada foi de acordo com o desenho do estudo utilizado e com o tipo de distribuição apresentado pelo conjunto dos dados analisados pelos testes estatísticos: Teste de Associação Exato de Fisher, análise de Correlação de Pearson, teste não paramétrico Kruskal-Wallis, teste não paramétrico Mann Whitney. Os achados da pesquisa tiveram suas informações cruzadas a fim de traçar o perfil desta amostra. Os resultados foram considerados significativos a um nível de significância máximo de $5 \%(p<0,05)$ e o software utilizado para esta análise foi o Statistical Package for Social Sciences (SPSS) versão 13,0 .

\section{RESULTADOS}

A amostra foi composta por 31 mulheres entre 21 e 70 anos que concluíram a avaliação por meio de três questionários. Na Tabela 1 apresenta-se a ca- racterização socioeconômicas da amostra investigada. Em relação ao estado civil, a maioria das mulheres casadas $(58,1 \%)$, destacando 6 professoras $(19,4 \%)$ e 4 médicas (12,9\%), com apenas 1 delas do $\operatorname{lar}(3,2 \%)$ e as outras 30 mulheres são profissionalmente ativas (96,8\%). A faixa etária mais encontrada com 12 mulheres foi a de mais de 50 anos de idade $(38,7 \%)$, seguida de 10 mulheres com menos de 40 anos de idade (32,3\%). Já a renda familiar apresentou, em maior prevalência, 9 mulheres acima de 15 salários mínimos (29\%) e 7 mulheres demonstraram ter renda abaixo de 4 salários mínimos (22,6\%).

Tabela 1. Características descritivas da população

\begin{tabular}{|c|c|c|c|}
\hline Variável & Resposta & $\mathrm{n}$ & $\%$ \\
\hline \multirow[t]{3}{*}{ Idade } & $20-40$ & 10 & 32,3 \\
\hline & $40-50$ & 9 & 29,0 \\
\hline & $50-70$ & 12 & 38,7 \\
\hline \multirow[t]{3}{*}{$\begin{array}{l}\text { Estado } \\
\text { Civil }\end{array}$} & Casada & 18 & 58,1 \\
\hline & Solteira & 5 & 16,1 \\
\hline & Outros & 8 & 25,8 \\
\hline \multirow[t]{6}{*}{ Renda } & Abaixo de 4 salários mínimos & 7 & 22,6 \\
\hline & Entre 4 e 6 salários mínimos & 4 & 12,9 \\
\hline & Entre 6 e 8 salários mínimos & 2 & 6,5 \\
\hline & Entre 8 e 10 salários mínimos & 4 & 12,9 \\
\hline & Entre 10 e 15 salários mínimos & 5 & 16,1 \\
\hline & Acima de 15 salários mínimos & 9 & 29,0 \\
\hline
\end{tabular}

anos. $n$ - número de mulheres em cada variável analisada.

Dezessete mulheres $(54,8 \%)$ praticavam outra atividade física antes de iniciar a prática de Pilates contra outras 14 mulheres (45,2\%) que não praticavam nenhuma atividade antes de praticar Pilates. Como resultado de frequência semanal obtivemos a maioria de 2 vezes por semana em 25 (80,6\%) muIheres, seguido de 1 vez por semana com $5(16,1 \%)$ mulheres.

Para avaliação de mudanças percebidas após o início da prática de Pilates, 14 mulheres $(45,2 \%)$ disseram que perceberam "Mudança no vigor e disposição do dia-a-dia", seguidas por 13 mulheres $(41,9 \%)$ que disseram que tiveram melhora no "Alívio de dores". 
O motivo pela procura da prática de Pilates em 12 mulheres $(38,7 \%)$ foi como uma forma "Terapêutica para melhorar algum acometimento em saúde", já 9 mulheres relataram que foi por "Recomendação médica" $(29,0 \%)$ e outras 9 mulheres optaram como forma de "Praticar uma atividade física e sair do sedentarismo", e apenas 1 optou como "Finalidade estética".

Quanto ao uso de medicação entre as voluntárias deste estudo, 10 (32,3\%) disseram que usam, enquanto $21(67,7 \%)$ responderam que não fazem uso de medicamentos.

Numa faixa etária entre 21 e 70 anos de idade, verificou-se uma média de $47,4 \pm 13$ anos, e uma média de tempo de prática de $2,9 \pm 3,1$ anos com o mínimo de 0,2 (2) meses e o máximo de 11 anos de prática.

Em relação aos domínios do questionário qualidade de vida (SF-36), não identificou nenhuma correlação significativa entre os achados. As respostas apesar de objetivas, seus descritores são subjetivos em intensidade de percepção sobre a saúde de forma geral, da qual foi descrita entre $14(45,2 \%)$ mulheres como "Muito Boa", 8 (25,8\%) descreveram como "Excelente" e 9 (29\%) como "Boa". Para descrever a comparação desta mesma percepção em relação há um ano atrás, 12 mulheres $(38,7 \%)$ responderam "Um pouco melhor" e "Muito melhor", apenas 1 (3,2\%) classificou como "Pior" e $6(19,4 \%)$ como "Quase a mesma".

A tabela 2, mostra as médias e os desvios-padrão obtido no SF-36 sendo que a maior foi $85,48 \pm 17,41$ para o domínio "Aspectos Sociais", enquanto o menor valor foi para "Vitalidade" 41,13 $\pm 8,73$.

Tabela 2. Estatísticas descritivas de Qualidade de Vida (SF-36)

\begin{tabular}{l|l|l|l}
\hline Domínios & $\mathrm{n}$ & Média & D.P. \\
\hline Capacidade funcional & 1 & 77,96 & 22,11 \\
\hline Limitação por aspectos físicos & 3 & 75,81 & 31,94 \\
\hline Dor & 3 & 69,06 & 21,95 \\
\hline Estado geral de saúde & 3 & 57,17 & 15,48 \\
\hline Vitalidade & 3 & 41,13 & 8,73 \\
\hline Aspectos sociais & 3 & 85,48 & 17,41 \\
\hline Limitação por aspectos emocionais & 3 & 78,49 & 30,49 \\
\hline Saúde mental & 3 & 69,55 & 12,76 \\
\hline
\end{tabular}

Resultados obtidos dentro dos domínios, onde D.P. = Desvio-Padrão.
Os domínios que tiveram os valores zerados foram "Limitações por aspectos emocionais" e "Limitação por aspectos físicos". Os valores máximos (100) foram alcançados para os domínios "Capacidade funcional", "Dor", "Limitação por aspectos físicos", "Limitação por aspectos emocionais" e "Aspectos sociais". As médias gerais, em ordem decrescente, para "Limitação por aspectos emocionais" $(78,49 \pm 30,49)$; "Capacidade Vital" $(77,96 \pm 22,11)$; "Limitação por aspectos Físicos" $(75,81 \pm 31,94)$; "Saúde Mental" $(69,55 \pm 12,76)$; "Dor" $(69,06 \pm 21,95) ; "$ "Estado de saúde" $(57,17 \pm 15,48)$.

Através dos resultados da Análise de Correlação, verificou-se que Idade, Frequência e Tempo de Pilates não apresentaram correlação significativa com os domínios do questionário SF-36.

A presença de estresse investigada no ISSL (Tabela 3), revela a percepção do indivíduo para o estresse experimentado no período do último mês (Quadro 3), na última semana (Quadro 2) e nas últimas 24 horas (Quadro 1). Nesta amostra foi observado apenas a percepção de estresse experimentado na última semana (Quadro 2) em 7 mulheres (22,6\%). Apesar da presença de estresse na última semana, não foi observada correlação significativa com as variáveis sócio-econômicas deste estudo.

Tabela 3. Estatística descritiva da Escala de Estresse (ISSL) percebido

\begin{tabular}{l|l|c|c}
\hline Escala stress & Resultado & $\mathrm{n}$ & $\%$ \\
\hline Quadro 1 & Ausência de stress & 31 & 100,0 \\
\hline & Presença de stress & - & - \\
\hline Quadro 2 & Ausência de stress & 24 & 77,4 \\
\hline & Presença de stress & 7 & 22,6 \\
\hline Quadro 3 & Ausência de stress & 31 & 100,0 \\
\hline & Presença de stress & - & - \\
\hline
\end{tabular}

Mostra a percepção do estresse experimentado nos períodos avaliados: Quadro 1 - nas últimas 24 horas, Quadro 2 - na última semana e Quadro 3 - no último mês.

\section{DISCUSSÃo}

As características de praticantes de Pilates mais recorrentes deste estudo foram mulheres de meia idade, casadas, com renda familiar acima de quinze 
salários mínimos, profissionalmente ativas. Mulheres tem maior nível de morbidade e fatores socioeconômicos influenciam na saúde além de idade, sexo e renda familiar que podem afetar o interesse do consumidor para determinados serviços, e tais descritores tornam-se relevantes para os profissionais entenderem seus consumidores, atrair e fidelizar seus clientes [17-20].

Atualmente, a valorização por estilos de vida mais ativo, tem despertado a prática de Pilates principalmente no público feminino e a preferência de duas vezes na semana foi observado mesmo entre aquelas que praticavam outra forma de atividade física antes do Pilates, e geralmente é a frequência de intervenção predominante nos trabalhos científicos com o método na literatura $[3,21]$.

O local da prática de Pilates é apontado por atividade de treinamento especializado capaz de abranger indivíduos com diferentes necessidades, seja na reabilitação ou para o condicionamento físico, tal prática, estreitam as relações de saúde e bem estar com benefícios além de físicos, emocionais, cognitivos e como respostas obtivemos melhora no vigor e na disposição do dia-a-dia, além do alívio de dores como percepção pessoal após o início da prática. Santos (2006) [22] sugeriu que as mudanças na autoestima, é uma percepção positiva, assim como, o vigor e a disposição, e podem estar associadas com a percepção de melhora do condicionamento físico, mais do que com as mudanças reais no condicionamento físico $[23,24]$.

A palavra "Saúde" é tratada muitas vezes, como sendo uma dimensão motivacional capaz de incentivar e fidelizar as pessoas a novos hábitos como a prática de atividade física, conforme observado neste estudo em que o motivo pela prática preferencialmente foi "Melhorar algum acometimento em saúde" seguido por "Sair do sedentarismo" e por "Recomendação médica", e são apreciadas como integrantes de qualidade de vida e saúde. Queixas de dor são geralmente motivo de realização de uma abordagem terapêutica do método Pilates e são comuns entre os que optam por saúde ao iniciarem com a prática do método [7].

Aptidão física, saúde e disposição foram os principais motivos encontrados no estudo de Fermino e colaboradores (2010) [11] em resultados referente a prática em academia, e corroboram com os resultados de Gonçalves (2015) [19] entre praticantes de Pilates, assim como os nossos achados, deixando subentendido que trata-se da prática de uma atividade física com benefícios em saúde independente da modalidade.

A literatura traz o que é observado na prática destas mulheres, onde o método Pilates é capaz de beneficiar, satisfazer e atender as necessidades de um público diversificado que busca "promoção da saúde" independente de doenças crônicas [21,22]. Em nossos resultados, achamos quanto ao uso de medicação que a maioria nesta amostra não faz uso, mesmo que sejam poucas as mulheres que não possuem acometimentos de doenças crônicas, corroborando com os achados de que a prática de atividade física pode resultar em redução do uso de medicação conforme cita Ribeiro e Moreira (2010) [25] em pacientes com lombalgia crônica após 10 sessões de exercícios.

A necessidade pela prática de atividade física nasce de um desconforto das condições físicas ou psicológicas pessoais para iniciar a prática de Pilates, o que foi mostrado por Gonçalves e colaboradores (2015) [19] em sua amostra de indivíduos com uma média de 46,9 anos de idade em uma fase de vida ativa e mais predisposta ao estresse, semelhante aos nossos achados.

Já a permanência do praticante, está interligada as percepções pessoais dos benefícios psicológicos e mentais, sobre o aspecto físico que somam-se também as necessidades desta prática por causa da qualidade de vida, como observamos em nossos achados $[22,24,26]$.

A percepção em geral da saúde pessoal foi "Muito boa" pela maioria das participantes. Quando comparado há um ano atrás, os relatos que mais aparecem são: "Muito melhor" e "Um pouco melhor", o que condiz com uma auto interpretação positiva da qualidade de vida, que pode estar associada com um programa continuado de Pilates [7]. Machado (2003) [27] fala que sensações de satisfação e prazer são geradas pelos movimentos do corpo com o Pilates, e afetam e são afetadas, pela motivação da prática regular que consequentemente pode ter sido a cau- 
sa e o motivo que levaram a persistência pela prática do Pilates observada neste estudo com a média de 2,9 anos de tempo de prática entre estas praticantes da amostra.

Com o passar dos anos há uma queda fisiológica progressiva que se inicia aos 25 anos e se acentua aos 60 anos com restrições ao movimento, resistência, equilíbrio, força e processos cognitivos que podem não traduzir um bem estar com qualidade de vida e tais indicadores podem ser avaliados por medidas de satisfação como o questionário de qualidade de vida [28]. Apesar dos benefícios, existem algumas limitações de tais instrumentos, que por serem compostos de perguntas fechadas e respostas objetivas que limitam-se as opções disponibilizadas [19].

O estresse envolve fatores físicos e psicológicos que são contrários à uma melhor qualidade de vida. Um estudo mostrou que o Pilates representa uma prática positiva, mas que pode ser uma experiência frustrante se influenciar de forma negativa a rotina dos praticantes [7]. Corroborando com isto, nosso estudo observou a presença de estresse principalmente em sintomas psicológicos, caracterizados pela persistência do estímulo estressor que não foi devidamente controlado e ter passado da primeira fase (Alerta), sendo experimentado e percebido nas últimas semanas (Fase de Resistência) conforme resultados do ISSL [29].

Uma ampla faixa etária (21 a 70 anos) pode ter influenciado na correlação com as variáveis tempo de prática, frequência semanal de Pilates, presença de estresse e qualidade de vida. As idades representam características individuais relevantes, e conforme Santos (2006) [22] indivíduos fisicamente ativos tem sua idade biológica reduzida em relação ao indivíduo sedentário, gerando disposição e vigor, conforme observado nos relatos das praticantes desta amostra após o início da prática de Pilates.

A presença do estresse nesta amostra, tem como perfil mais recorrente serem mulheres casadas com idade abaixo de 40 anos, renda familiar abaixo de 4 salários mínimos e frequência semanal de 2 vezes por semana de Pilates. $O$ somatório de tais características condizem com as percepções pessoais de estresse onde as constantes pressões do dia-a-dia resultam em prejuízos ao bem estar e saúde [27].

Escores próximos do ideal (100 pontos), obtidos neste estudo, são achados positivos para os domínios do SF-36 que caracterizam uma boa qualidade de vida. E soma-se ao resultado da principal mudança observada após a prática de Pilates, relatada por estas praticantes com "Melhora no vigor" e mesmo sendo sinônimo de Vitalidade, uma maioria apresentou resultados com baixas pontuações no SF-36 neste domínio. Sugere-se que haja pontos de vista divergentes para os mesmos aspectos, mas que podem ser explicados pelas poucas alternativas de escolhas do questionário fechado, empregado neste estudo, como fator limitante entre estes achados.

Em nossa amostra, poucas mulheres tem a presença do estresse e somada com o achado do domínio "Vitalidade" do questionário de qualidade de vida, sugere que ambas as informações se complementem, pois referem-se a algo percebido pelo indivíduo a respeito de energia, disposição, cansaço e esgotamento.

Vitalidade é o que envolve as habilidades de realizar as atividades cotidianas com mais disposição. A idade é uma variável importante que prediz as expectativas, necessidades e ideais de cada fase de vida do indivíduo e nesta amostra independente da faixa etária, o domínio Vitalidade manteve-se com pontuações mais baixas em relação aos demais domínios $[27,30]$.

O retorno a funcionalidade é um dos objetivos primários do método Pilates e tem estreita relação com a disposição do dia-a-dia, e quando este objetivo é alcançado, ele é capaz de refletir nosso bem estar físico e mental [31]. Este aspecto foi observado entre as mulheres que não possuem acometimentos na coluna vertebral, pois as pontuações obtidas no questionário qualidade de vida estão mais elevadas, mesmo no domínio Vitalidade.

Geneen e colaboradores (2017) [32] observaram que a prática de atividade física em indivíduos acometidos por doença crônica trazem poucos eventos adversos e contribuem positivamente para a melhora na gravidade da dor e na qualidade de vida, assim como em nossos achados. 


\section{CONCLUSÃO}

Percebe-se que a prática de Pilates pode influenciar positivamente no controle de níveis baixos de estresse percebido, por meio dos resultados demostrados em nosso estudo nos questionários "Inventário de Sintomas de Estresse para Adultos de LIPP" (ISSL), "Questionário Qualidade de vida" (SF-36) e também no "Questionário de prática de Pilates". Sendo assim, nosso estudo pode contribuir para o desenvolvimento e direcionamento de estratégias adequadas as percepções dos diferentes praticantes, principalmente, para os profissionais que buscam na qualidade do atendimento propiciar o bem estar a seus clientes.

\section{REFERÊNCIAS}

Butelli ACK; Costa RR; Kruel LF. Efeitos do método Pilates no comportamento do perfil lipídico de mulheres sedentárias: uma revisão narrativa. Rev Arq de Ciênc Saúde. 2015;22(4):9-13.

2. Di Lorenzo EC. What is it? Should it be used in rehabilitation? Sports Health. 2011;3(4):352-361.

3. Silva ACLG, Mannrich G. Pilates na reabilitação: uma revisão sistemática. Fisioter Mov. 2009;22(3):449-455.

4. Latey P. The pilates method: history and philosophy. J Bodyw Mov Ther. 2001;5(4):275-282.

5. Ciconelli RM, Ferraz MB, Santos W, Meinão I, Quaresma MR. Tradução para a língua portuguesa e validação do questionário genérico de avaliação de qualidade de vida SF-36 (Brasil SF-36). São Paulo: Universidade Federal de São Paulo;1997.

6. Mcneill W. Decision making in Pilates. J Bodyw Mov Ther. 2011;15(1):103-107.

7. Arsego NR. Motivos que levam a prática regular de atividades físicas: Um estudo com praticantes de ginástica de academia. Porto Alegre: Universidade Federal do Rio Grande do Sul; 2015.

8. Natour J, Cazotti LA, Ribeiro LH, Baptista AS, Jones A. Pilates improves pain, function and quality of life in patients with chronic low back pain: a randomized controlled trial. Clin Rehabil. 2015;29(1):59-68.

9. Bittencourt JR. O método Pilates nas páginas dos jornais: saúde, consumo, boa forma, mulheres. Porto Alegre: Universidade Federal do Rio Grande do Sul; 2015.
10. Gandek M, Montazeri A, Goshtasebi A, Vahdaninia M. The short form health survey (SF-36): Translation and validation study of the Iranian version. Quality of Life Research 2004;14(3):875-882.

11. Fermino RC, Pezzini MR, Reis RS. Motivos para a prática de atividade física e imagem corporal em frequentadores de academia. Clínica Médica do Exercício e do Esporte. 2010;16(1):18-23.

12. Coelho VJB. Características psicográficas no consumo do fitness em ginásios de mulheres. Lisboa, Portugal. Universidade Técnica de Lisboa, 2009.

13. Amorim SS, Nascimento MC, Andrade A, Carvalho T. Avaliação da qualidade de atendimento na percepção dos participantes de programa estruturado de exercício físico, com levantamento do perfil dos praticantes. Rev Bras Atividade Física e Saúde. 2002;7(3):4-11.

14. Gonçalves CR. Retenção de sócios no fitness: Estudos do posicionamento, expectativas, bem-estar e satisfação. Lisboa, Portugal. Universidade Técnica de Lisboa, 2012.

15. Oliveira RGO, Oliveira JNRF, Silva PR, Oliveira LC, Fernandes JAA, Oliveira RF. et al. A influência do método Pilates para as praticantes. Rev Bras Ciênc e Mov. 2015; 23(3):47-52.

16. Lipp MEN, Guevara AJH. Validação empírica do Inventário de Sintomas de Stress (ISS). Est de Psicologia. 2000;11,43-49.

17. Buchmann CB. Análise da qualidade de serviços: Percepções de consumidores na faixa etária de 30 a 38 anos em academias de ginástica de Porto Alegre. Universidade Federal do Rio Grande do Sul, Porto Alegre. 2015.

18. Kaleta D, Polanska K, Zaborszczk DE, Wojciech H, Ddrygas W. Factors influencing self-perception of health. Cent Eur J Public-Health. 2009;17(3):122-127.

19. Gonçalves NS, Silva RP, Costa-Silva RA, Junqueira LMB, Sousa LE. Motivos para a prática de Pilates em Mariana. Rev Bras de Ciências da Saúde. 2015;19(4):277-284.

20. Saba F. Gestão em Atendimento: Manual prático para academias e centros esportivos. São Paulo: Manole, 2012.

21. Lessen D, Anderson E. The Pilates Method Alliance Certification exam study guide. Miami: PMA, 2006.

22. Santos SC, Knijnik JD. Motivos de adesão à prática de atividade física na vida adulta intermediária I. Revista Mackenzie de Educação Física e Esporte. 2006;5(1):23-24.

23. Vieira FT, Faria LM, Wittmann JI,Teixeira W, Nogueira LA. The influence of pilates method in quality of life of practitioners. J Bodyw Mov Ther. 2013;17(4):483-487. 
24. Byrnes K, Wu P-J, Whillier S. Is Pilates an effective rehabilitation tool? A systematic review. J Bodyw Mov Ther. 2010;23(5):24-27.

25. Ribeiro C, Moreira D. O exercício terapêutico no tratamento da lombalgia crônica: uma revisão da literatura. Rev Bras Cienc e Mov. 2010;18(4):100-108.

26. Küçük F, Livanelioglu A. Impact of the clinical pilates exercises and verbal education on exercise beliefs and psychosocial factors in healthy women. J Phys Ther Sci. 2015;27(11):3437-3443.

27. Machado SS. Qualidade de vida e estresse de adultos jovens na sociedade contemporânea. Porto Alegre: Universidade Federal do Rio Grande do Sul. 2003.

28. Gonçalves SP, Lima POP. Percepção de saúde e qualidade de vida: um inquérito com praticantes de Pilates. Rev Fisiot S Fun. 2014;3(1):11-17.
29. Stults-Kolehmainen MA, Sinha R. The Effects of Stress on Physical Activity and Exercise. Sports Med. 2014;44(1):81-121.

30. Küçük F, Poyraz BK, Idiman E. Improvements in cognition, quality of life, and physical performance with clinical Pilates in multiple sclerosis: a randomized controlled trial. J Phys Ther Sci. 2016;28(3):761-768.

31. Botelho N, Lipp MEN, Manso PG, Furlanetto R, Abreu MT. Stress and depression in graves ' disease. Pinnacle Medicine \& Medical Sciences. 2014;1(3):309-314.

32. Geneen J, Moore RA, Clarke C, Martin D, Colvin LA, Smith LH. Physical activity and exercise for chronic pain in adults: an overview of Cochrane Reviews. Cochrane Database Syst Rev. 2017;24(4). 